\title{
Effects of the COVID-19 pandemic on reperfusion therapy for acute ischemic stroke patients in Huizhou City, China
}

\author{
Weiliang Luo ${ }^{1}$ (D) Jiming $\mathrm{Li}^{1} \cdot{\text { Zhuqin } \mathrm{Li}^{1} \cdot \text { Xuanwen Luo }}^{1} \cdot$ Minrui Chen ${ }^{2} \cdot$ Chunsheng Cai $^{3}$
}

Received: 15 September 2020 / Accepted: 23 November 2020 / Published online: 7 January 2021

(C) Fondazione Società Italiana di Neurologia 2021

\begin{abstract}
Background and purpose The COVID-19 pandemic has impacted the reperfusion therapy for acute ischemic stroke (AIS) patients. Huizhou City utilized its experience with the SARS and MERS breakouts to establish a reperfusion treatment program for AIS patients.

Method This is a retrospective study on 8 certified stroke hospitals in Huizhou City from January 2020 to May 2020. We analyzed the number of AIS patients with reperfusion therapy, stroke type (anterior/posterior circulation stroke), modes of transport to hospital, NIHSS score, onset to door time (ODT), door to needle time (DNT), and door to puncture time (DPT). The analysis was compared with baseline data from the same time period in 2019.

Result In 2020, the number of AIS patients receiving reperfusion therapy decreased (315 vs. 377), NIHSS score increased $[8(4,15)$ vs. $7[(1,2)], P=0.024]$, ODT increased $[126(67.5,210.0)$ vs. $120.0(64.0,179.0), P=0.032]$, and DNT decreased $[40(32.5,55)$ vs. $48(36,59), P=0.003]$. DPT did not change. Seventy percent of AIS patients indicated self-visit as their main mode of transport to the hospital. In both periods, mild stroke patients were more likely to self-visit than utilize emergency systems [2019: 152 (57.6\%) vs. 20 (45.6\%), $P=0.034 ; 2020: 123$ (56.9\%) vs. 5 (14.7\%), $P<0.001]$. The NIHSS score for self-visiting patients was lower for patients who utilized the ambulance system in both years [self-visit: $6.00(3.00,12.00)$, ambulance: $14.00(9.00,19.00)$, $P<0.001]$. The volume of reperfusion patients was lower in 2020; however, the decrease was only significant $(P=0.028)$ in February 2020.

Conclusion During the COVID-19 pandemic in 2020, the number of AIS patients receiving reperfusion therapy significantly decreased when compared to the same period in 2019. The patients' condition increased severity, ODT increased, and the DNT decreased. DPT was not significant for self-visiting and ambulance patients. Moderate to severe stroke patients were more likely to utilize ambulance services.
\end{abstract}

Keywords Stroke $\cdot$ COVID-19 $\cdot$ Reperfusion

\section{Abbreviations}

AIS Acute ischemic stroke

NIHSS National Institute of Health Stroke Scale

Weiliang Luo and Jiming Li contributed equally to this work.

Weiliang Luo 1w1306@126.com

1 Department of Neurology, Huizhou Municipal Central Hospital, No. 41, Eling North Road, Huizhou 516001, Guangdong Province, People's Republic of China

2 Guangdong Medical University, Zhanjiang, Guangdong Province, People's Republic of China

3 Department of Neurology, Huizhou First Hospital, Huizhou, Guangdong Province, People's Republic of China

\author{
ODT Onset to door time \\ DNT Door to needle time \\ DPT Door to puncture time \\ BOSC Big Data Observatory Platform for stroke of China
}

Huizhou City is located in the southern part of Guangdong Province of China, with an area of $11,599 \mathrm{~km}^{2}$ and a population of approximately 5 million. In 2019, five hospitals in Huizhou were certified by The Stroke Prevention Project Commission of the National Health Commission in China to perform thrombolytic therapy for acute ischemic stroke (AIS) patients, and three hospitals were certified for both thrombolytic therapy and endovascular mechanical thrombectomy for AIS patients. Stroke patients within $24 \mathrm{~h}$ of onset were sent to the nearest one of these eight hospitals for reperfusion 
treatment. These eight hospitals were strategically located to be accessible throughout the city, forming a stroke emergency network in Huizhou. With improvements in road construction within the past 10 years, the eight service areas are all within an hour's drive.

The COVID-19 outbreak in Wuhan became a global public health emergency in January 2020 [3, 4]. On January 23, 2020, Guangdong Province initiated the first-level public health emergency response, and transitioned to the second-level response on February 24, 2020, through May 8, 2020. According to the State Council of the People's Republic of China on January 8, 2016, they publicly issued and implemented the "General Emergency Response Plan for National Emergencies" for the classification of public emergencies, the first-level response being the highest level response. Since Huizhou had experience with SARS in 2003 and had the experience of successfully curing the only MERS-CO case imported into mainland China in 2017 [ 1], the government immediately designated one hospital for the treatment and isolation of COVID-19 patients. This designated hospital is a stroke emergency hospital qualified for thrombolysis and thrombectomy. All hospitals established fever clinics to proactively screen for COVID-19 patients. The COVID-19 outbreak response was different than with SARS. The government's management was stricter as they implemented community and village lockdowns. Hospitals closed for non-essential services, restricted visitors, and restricted non-essential staff from entering the hospitals. For the safety of the patients and their families, staff, students, and volunteers, the hospitals formulated strict and effective guidelines for entering and leaving the hospitals. Patients, family members, and medical staff were all required to wear personal protective equipment.

During the pandemic, acute stroke remains as an emergency condition and needs to be treated immediately. The 8 certified hospitals in Huizhou continued to provide services. We compared the reperfusion treatment for AIS patients in the city between January and May 2020 with the same period in 2019, and analyzed the impact of the outbreak.

\section{Method}

The design of the study Data originated from low-risk original data and the third author (Z.Q.Lee) can be contacted if the relevant information is requested. The Institutional Review Board of Huizhou Municipal Central Hospital Affiliated to Guangdong Medical University approved this research protocol.

Data was collected from the 8 hospitals in Huizhou City certified by the Stroke Prevention Project Commission of the National Health Commission in China. Our study included patients who were admitted to any of the 8 certified stroke hospitals in Huizhou City and received reperfusion treatment (thrombolysis and mechanical thrombectomy). These 8 hospitals have dedicated data managers, and were required to report information monthly regarding patients with acute stroke thrombolysis and mechanical thrombectomy, such as the following: stroke type(anterior/posterior circulation stroke) [5], the number of stroke patients with thrombolysis and thrombectomy, the time from symptoms onset to arrival to hospital (onset to door time, ODT), the time of reperfusion treatment (door-to-needle, DTN, and door-to-puncture, DTP), the mode of transport to the hospital, the NIHSS score upon hospital arrival, and other data. The data was reported to the Big Data Observatory Platform for stroke of China (BOSC) through the network. BOSC was established in 2017 (https:// sinosc.org/home/index). Data collection began from January 1, 2020, to May 31, 2020, mirroring the start of the pandemic to the end of the second-level public health emergency response, and was compared with baseline data of the same period last year.

\section{Statistics and analysis}

This is a retrospective simple descriptive study. Data presentation uses the actual number or percentage. SPSS 19 statistical software was used for statistical analysis of the obtained data, chi-square $\left(\chi^{2}\right)$ statistic was used for data analysis, and non-parametric test was used for data measurement. All tests used a two-sided test $P<0.05$ was considered statistically significant.

\section{Result}

AIS admission among the 8 certified stroke hospitals in Huizhou City decreased by $16.6 \%$ in 2020 since 2019 (4369 vs. 5238). Although the total AIS admission in 2020 has decreased significantly compared with 2019 , the rate of AIS receiving reperfusion therapy in the same period of 2 years is very close (7.1\% in 2020 (315/4369), 7.2\% in 2019 (377/ 5238)). Comparison of the two timeframes, in terms of gender, age, wake-up stroke, the mode of transport to hospital ((a) self-visit; (b) call 120 ambulance; (c) transfer from outside hospital: refer from the hospital without qualification for reperfusion; (d) onset in the hospital), reperfusion method (intravenous thrombolysis, mechanical thrombectomy, bridging thrombolysis), intravenous thrombolysis whether with alteplase or urokinase, onset to needle time (ONT), and DPT, the differences were not statistically significant $(P \geq$ 0.05). However, the NIHSS score before treatment, ODT, and DNT were found to be statistically significant $(P<0.05)$ between both groups. According to the Oxford Community Stroke Classification, the percentage and total number of 
anterior and posterior circulation strokes in the same period of 2 years is not statistically significant [5]. (See Table 1).

The main mode of transport to the hospital for stroke patients was self-visiting, and both groups accounted for about $70 \%$. In 2019, the number of patients who arrive at the hospital through 120 ambulances was higher than that of outside hospital referrals ( 47 vs. $34, P=0.149$ ). However, in 2020, the utilization of 120 ambulances was lower than the referral rates from external hospitals (41 vs. $51, \mathrm{P}=0.149$ ), but the differences were not statistically significant (see Fig. 1). The proportion of mild stroke (NIHSS $<8$ points) patients who selfvisit to the hospital were higher than the patients who called 120 ambulances [2019: $152(57.6 \%)$ vs. $20(45.6 \%), P=$ $0.034 ; 2020$ : $123(56.9 \%)$ vs. 5 (14.7\%), $P<0.001]$. The NIHSS scores of the two comparative groups who self-visit to the hospital were significantly lower than the patients who called 120 ambulances [6.00 (3.00, 12.00), 14.00 (9.00, $19.00), P<0.001$ ] (see Table 2). In the 2020 group, the proportion of patients who called 120 ambulances with moderate to severe ischemic stroke were significantly higher than those who came to the hospital by themselves ( $82.4 \%$ vs. $60.1 \%$ ) (see Table 2 and Table 3). The ODT and ONT in the 2020 group are significantly higher than those of the 2019 group [ODT: $105.00(60.00-165.00)$ vs. 302.50 (167.50-617.75), $P=0.012$; ONT: $158.00(107.50-210.50)$ vs. 235.00 (235.00-235.00), $P=0.002$ ] (see Table 3 ).

The number of AIS patients with reperfusion treatment who came to the hospital during the peak outbreak of the epidemic in February 2020 was significantly lower than that in February 2019 (see Fig. 2). The number of AIS patients decreased at week 4 of 2020 and reached to the lowest level at week 10 and then increased; the declined period lasted for about 6 weeks, while in 2019, volume decreased from week 6 and to the lowest level at week 7 and then increased; the declined period lasted for about 1 week.

\section{Discussion}

COVID-19 has a tremendous impact on the treatment of patients with acute ischemic stroke. This study revealed that during the pandemic from January 01, 2020, to May 31, 2020, compared with the same period in 2019, AIS admission decreased by $16.6 \%$; also, the number of patients receiving

Table 1 Characteristics of patients with reperfusion treatment from Jan 1 to May 31 in 2019 and 2020

\begin{tabular}{|c|c|c|c|c|c|}
\hline & & $2019(n=377)$ & $2020(n=315)$ & Total $(N=692)$ & $P$ value \\
\hline Sex & $\begin{array}{l}\text { Female }(n, \%) \\
\text { Male }(n, \%)\end{array}$ & $\begin{array}{l}126(33.4 \%) \\
251(66.6 \%)\end{array}$ & $\begin{array}{l}97(30.8 \%) \\
218(69.2 \%)\end{array}$ & $\begin{array}{l}223(32.2 \%) \\
469(67.8 \%)\end{array}$ & 0.461 \\
\hline Age & & $64.30 \pm 12.57$ & $62.98 \pm 12.83$ & $63.70 \pm 12.70$ & 0.379 \\
\hline Wake-up stroke & $\begin{array}{l}\text { No }(n, \%) \\
\text { Yes }(n, \%) \\
\text { Miss }(n, \%)\end{array}$ & $\begin{array}{l}320(84.9 \%) \\
44(11.7 \%) \\
13(3.4 \%)\end{array}$ & $\begin{array}{l}277(87.9 \%) \\
38(12.1 \%)\end{array}$ & $\begin{array}{l}597(86.3 \%) \\
82(11.8 \%) \\
13(1.9 \%)\end{array}$ & 0.992 \\
\hline Mode of transport to hospital & $\begin{array}{l}\text { Self-visit }(n, \%) \\
\text { Call } 120 \text { ambulance }(n, \%) \\
\text { Transfer from outside hospital }(n, \%) \\
\text { Onset in the hospital }(n, \%) \\
\text { Miss }(n, \%)\end{array}$ & $\begin{array}{l}264(70.0 \%) \\
47(12.5 \%) \\
41(10.9 \%) \\
9(2.4 \%) \\
16(4.2 \%)\end{array}$ & $\begin{array}{l}216(68.6 \%) \\
34(10.8 \%) \\
51(16.2 \%) \\
13(4.1 \%) \\
1(0.3 \%)\end{array}$ & $\begin{array}{l}480(69.4 \%) \\
81(11.7 \%) \\
92(13.3 \%) \\
22(3.2 \%) \\
17(2.5 \%)\end{array}$ & 0.141 \\
\hline Reperfusion method & $\begin{array}{l}\text { Intravenous thrombolysis }(n, \%) \\
\text { Mechanical thrombectomy }(n, \%) \\
\text { Bridging thrombolysis }(n, \%) \\
\text { Miss }(n, \%)\end{array}$ & $\begin{array}{l}293(77.7 \%) \\
67(17.8 \%) \\
17(4.5 \%)\end{array}$ & $\begin{array}{l}231(73.3 \%) \\
70(22.2 \%) \\
13(4.1 \%) \\
1(0.3 \%)\end{array}$ & $\begin{array}{l}524(75.7 \%) \\
137(19.8 \%) \\
30(4.3 \%) \\
1(0.1 \%)\end{array}$ & 0.331 \\
\hline NIHSS score before treatment & Median (IQR) & $7(3,13)$ & $8(4,15)$ & $7(3,14)$ & 0.024 \\
\hline $\begin{array}{l}\text { Medicine of intravenous } \\
\text { thrombolysis }\end{array}$ & $\begin{array}{l}\text { None } \\
\text { Alteplase }(n, \%) \\
\text { Urokinase }(n, \%)\end{array}$ & $\begin{array}{l}60(15.9 \%) \\
276(73.2 \%) \\
41(10.9 \%)\end{array}$ & $\begin{array}{l}71(22.5 \%) \\
212(67.3 \%) \\
32(10.2 \%)\end{array}$ & $\begin{array}{l}131(18.9 \%) \\
488(70.5 \%) \\
73(10.5 \%)\end{array}$ & 0.950 \\
\hline Stroke type & $\begin{array}{l}\text { Anterior circulation }(n, \%) \\
\text { Posterior circulation }(n, \%)\end{array}$ & $\begin{array}{l}315(83.3 \%) \\
63(16.7 \%)\end{array}$ & $\begin{array}{l}268(85.4 .8 \%) \\
46(14.6 \%)\end{array}$ & $\begin{array}{l}583(84.2 \%) \\
109(15.8 \%)\end{array}$ & 0.530 \\
\hline ODT (min) & Median (IQR) & $120.0(64.0,179.0)$ & $126(67.5210 .0)$ & $120.0(64.0,199.0)$ & 0.032 \\
\hline DNT (min) & Median (IQR) & $48(36,59)$ & $40(32.5,55)$ & $44(34,58)$ & 0.003 \\
\hline ONT (min) & Median (IQR) & $160(106.75,212.25)$ & $157.5(103.75,211.75)$ & $158.5(106,212)$ & 0.791 \\
\hline DPT (min) & Median (IQR) & $135(82.5,209.5)$ & $118(89,149.25)$ & $128(87,179)$ & 0.118 \\
\hline
\end{tabular}

$O D T$, onset to door time; $D N T$, door to needle time; $O N T$, onset to needle time; $D P T$, door to puncture time 
Table 2 Comparison of transport mode to hospital for patients with reperfusion treatment from Jan 1 to May 31 in 2019 and 2020

\begin{tabular}{llccc}
\hline & & Self-visit & Call 120 ambulance & $P$ value \\
\hline $\begin{array}{l}\text { NIHSS score before } \\
\text { treatment }\end{array}$ & Median (IQR) & $6.00(3.00,12.00)$ & $14.00(9.00,19.00)$ & 0.000 \\
ODT & Median (IQR) & $105.00(60.00,165.00)$ & $302.50(167.50,617.75)$ & 0.012 \\
DNT & Median (IQR) & $44.00(34.00,58.00)$ & $86.75(51.00,122.50)$ & 0.518 \\
ONT & Median (IQR) & $158.00(107.50,210.50)$ & $235.00(235.00,235.00)$ & 0.002 \\
DPT & Median (IQR) & $150.00(60.00,310.50)$ & $116.00(79.00,170.50)$ & 0.127 \\
\hline
\end{tabular}

$O D T$, onset to door time; $D N T$, door to needle time; $O N T$, onset to needle time; $D P T$, door to puncture time reperfusion treatment among the 8 certified stoke emergency hospitals in Huizhou decreased by about $1 / 6$ (16.4\%), of which the number of intravenous thrombolysis decreased by $21.2 \%$, and the number of mechanical thrombectomy decreased by $1.2 \%$. In January 2019 , due to the traditional Chinese Spring Festival, people prefer not to visit the hospital for medical care during this period. Therefore, the number of AIS reperfusion treatments became the lowest in 5 months, and then the number began to increase month by month. The number of AIS reperfusion patients admitted in January 2020 increased compared to the same period in 2019. However, due to the first-level public health emergency response and the Spring Festival, the number of AIS reperfusion patients decreased sharply during week 3 in January 2020. The number of reperfusion patients admitted in February 2020 decreased by $48.8 \%$ (21 vs. 41 ) compared with the same period in 2019 , and the number of mechanical thrombectomy patients decreased by $6.7 \%$ (14 vs. 15) (see Figs. 1 and 3). The number of intravenous thrombolysis decreased close to $50 \%$ during the peak outbreak in February 2020 compared to 2019, and it is similar to the report from Shanghai, China [6]. The number of mechanical thrombectomy in February reported by 280 hospitals in China's BOSC system decreased by $25.3 \%$ compared to 2019 in the same period, and the number of intravenous thrombolysis decreased by $26.7 \%$ [7].

In other regions and countries with COVID-19 outbreaks, the number of hospitalized AIS patients has decreased to varying degrees [8]. Our data analysis, supported by literature review, found that the decrease in patient volume is mainly caused by the stay-at-home order which kept mild patients at home, social isolation which inadvertently delayed stroke detection, increased emergency cases which resulted in stroke patients directed to non-stroke facilities, comorbidities masking stroke symptoms which caused misdiagnoses of AIS, and external changes in environmental and behavioral factors which may have reduced AIS risk [9]. Compared with other regions in mainland China, the reduction rate of thrombectomy in patients with acute ischemic stroke in Huizhou is not obvious (only 1.2\%), and this may be that due to the experience of reperfusion therapy for acute ischemic stroke while in combating SARS and MERS, Huizhou did not stop emergency stroke services in the early stage of the outbreak of COVID-19. Experience in combating SARS and MERS taught medical staff, patients, and their families that simple protective measures such as wearing surgical masks and frequent hand washing can effectively prevent the spread of disease [10]. According to the literature, to perform a CT scan of the lungs at the same time to perform a CT scan of the head for patients with suspected strokes can help to rule in or rule out COVID-19. The imaging technicians have to protect themselves with wearing protective gowns, goggles, gloves, and surgical masks. The designated hospital for COVID-19 is equipped with a special CT machine to examine suspected and confirmed patients of COVID-19. If a CT scan revealed ground glass-like changes in the lungs and/or decreased blood routine lymphocytes, then COVID-19 is highly suspected [11,
Table 3 Comparison of transport mode to hospital for AIS patients with reperfusion treatment by severity from Jan 1 to May 31 in 2019 and 2020

\begin{tabular}{lccccc}
\hline & $2019(n, \%)$ & & \multicolumn{2}{c}{$2020(n, \%)$} & \\
\cline { 2 - 3 } \cline { 5 - 6 } \cline { 5 - 6 } & Self-visit & Call 120 ambulance & & Self-visit & Call 120 ambulance \\
\hline Total & 264 & 47 & 216 & 34 \\
Mild AIS (NIHSS < 8) & $152(57.6 \%)$ & $20(45.6 \%)$ & & $123(56.9 \%)$ & $5(14.7 \%)$ \\
Moderate AIS (NIHSS 8-15) & $47(17.8 \%)$ & $14(29.8 \%)$ & & $57(26.4 \%)$ & $17(50.0 \%)$ \\
Severe AIS (NIHSS > 15) & $31(11.7 \%)$ & $10(21.3 \%)$ & & $32(14.8 \%)$ & $11(32.4 \%)$ \\
Miss & $34(12.9 \%)$ & $3(6.4 \%)$ & & $4(1.8 \%)$ & $1(2.9 \%)$ \\
$P$ value & 0.034 & & $<0.001$ & \\
\hline
\end{tabular}

AIS, acute ischemic stroke 


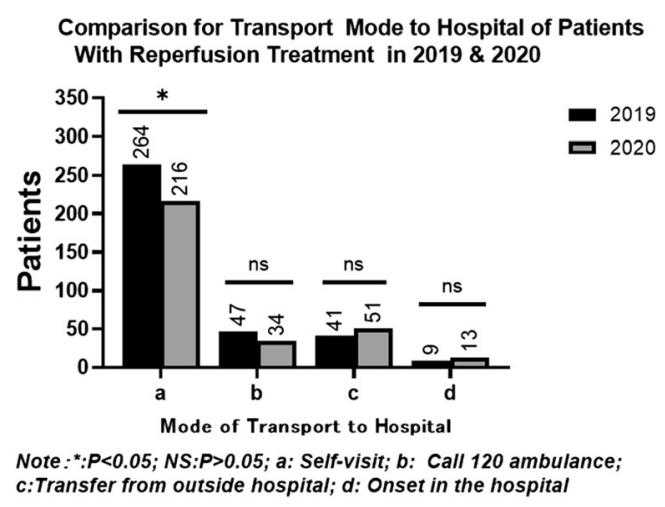

Fig. 1 Comparison for transport mode to hospital of patients with reperfusion treatment in 2019 and 2020

12], and a member of the COVID-19 expert team in the hospital will be consulted immediately; if the expert suspected COVID-19, then the patients were transferred to the isolation ward of the designated hospital for observation. For patients who are not suspected of COVID-19, they stayed in a single room for the first 3 days under observation and waited for the COVID-19 nucleic acid test results. These regulations are consistent with the guidelines on stroke treatment during COVID-19, issued by the Guangdong Provincial Cerebrovascular Disease Branch and the Health Commission of Mainland China [13].

Sixty-two patients with COVID-19 were treated in Huizhou City from January to May 2020, and all were cured and discharged by March 3, 2020. Luckily, patients with COVID-19 did not comorbid with acute stroke during the treatment period, and no medical staff was infected by COVID-19. Therefore, the number of patients with acute ischemic stroke for thrombectomy in 2020 has not decreased significantly compared with the same period in 2019. Huizhou City lifted the first-level public health emergency response in February 24, 2020, and the number of patients with acute stroke reperfusion treatment has shown a monthly increase.

Reports from southern Europe reported that AIS patients who were treated in 2020 were older and more severely ill, had longer ODT, and were more likely to call an ambulance

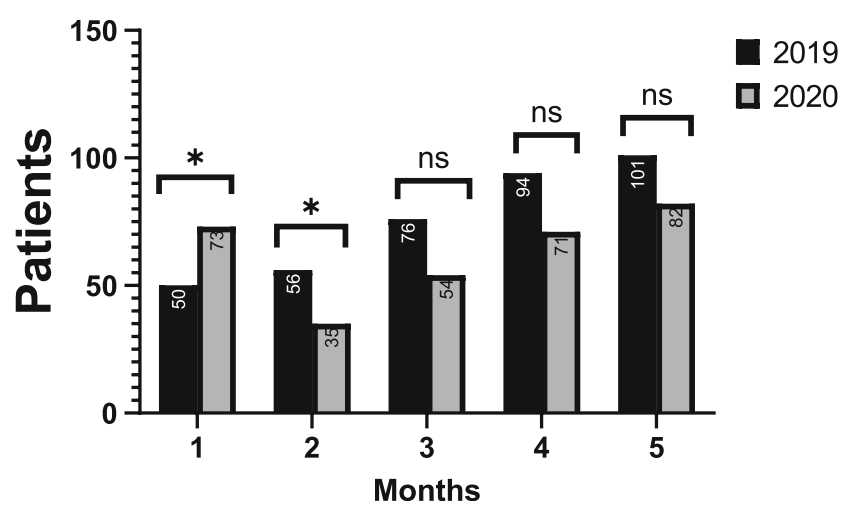

Fig. 2 The number of AIS patients with reperfusion treatment from Jan to May in 2019 and 2020

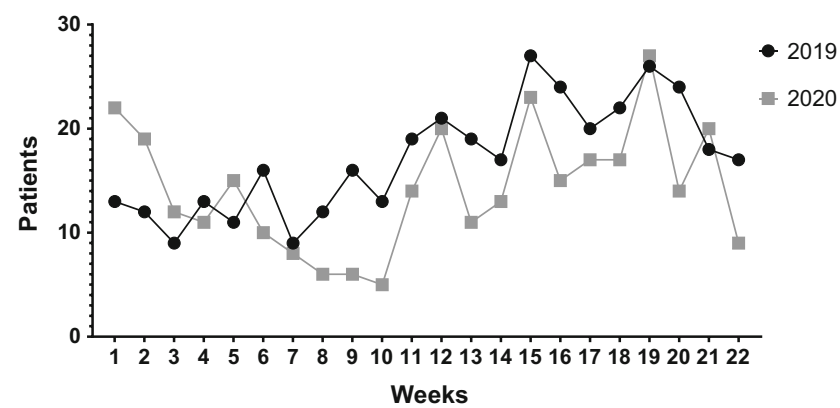

Fig. 3 The trend of AIS patients with reperfusion treatment by week in 2019 and 2020

for hospital visits due to fear of COVID-19 infection [8]. This study from our data found that there was no statistically significant difference in the age of the patients who visited the hospital from January to May 2020 compared to the same period in 2019.

Patients with mild symptoms were more reluctant to hospital visits, possibly due to fear and uncertainty. Simultaneously, public health efforts (such as restricting transportation, administrative reporting, and geographic restriction) may have inadvertently caused more inconvenience for patients to seek medical attention. Therefore, patients with mild symptoms often chose to observe at home. Patients with severe symptoms were more likely to seek medical care.

In Huizhou, the majority of patients with mild to moderate acute ischemic stroke were more likely to travel to the hospital using their own mode of transportation. This is due to the improvement of public transportation in China as well as the rate of car ownership. Most people in Huizhou were apprehensive with calling an ambulance due to uncertain wait time as well as fear in contracting the COVID-19 virus due to improper disinfectant protocols. We found out that most of the patients experienced anterior circulation strokes, we consider there being no COVID-19 impact on the stroke type.

However, patients with severe illness prefer to choose 120 rescue systems; the difference is more obvious especially during the impact of the COVID-19 pandemic. Patients with severe acuity believed that the 120 rescue systems could provide faster and more efficient medical care. In support, the ONT/ ODT showed to be significantly shorter for patients who utilized the 120 rescue systems than those who came to the hospital on their own. Having the option to call rescue systems encourages families and caretakers to be more attentive and allows them to initiate medical care more efficiently.

Guangdong Province, China, executed the first-level public health emergency response on January 23, 2020 (week 5 of the year), and simultaneously, reperfusion therapy for patients with acute ischemic stroke began to decline week by week. At the same time, Huizhou City started to admit COVID-19 patients in the designated hospital. All COVID-19 patients were cured and discharged on March 03, 2020 (week 9 of the year). Huizhou City began to increase the number of patient visits 
gradually since reaching the lowest during week 10 . The number of COVID-19 patients in the hospital impacted the number of AIS patient visits significantly. The number of AIS patient visits began to gradually increase only after all COVID-19 patients were completely cured and discharged. However, the number of patient visits per week was still less than during the same period in 2019. This suggests that the psychological fear and uncertainty related to the outbreak still exists. In 2020, the prevention and control cycle of COVID-19 in Huizhou City began to reverse the trend of the pandemic by fivefold. None of the acute stroke patients was suspected of or diagnosed with COVID-19 during their treatment period. In addition, no medical providers were infected with COVID-19 during the 2020 time period. This achievement was heavily dependent on the city's strict population movement control.

According to Chinese Guidelines for diagnosis and treatment of acute ischemic stroke, patients with acute ischemic stroke with an onset of $>4.5 \mathrm{~h}$ and $<6 \mathrm{~h}$ can be given urokinase intravenous thrombolytic therapy [2], but the usage of urokinase thrombolysis is very low. This study revealed that the DNT average time of thrombolytic therapy for acute ischemic stroke during the January to May 2020 outbreak in Huizhou City was $40 \mathrm{~min}$, an average decrease of $8 \mathrm{~min}$ compared with the same period in 2019, and the difference was statistically significant $(P<0.05)$. In comparison with the results of the significant prolonged DNT and DPT in southern European during the pandemic reported by Montaner et al. [8], the DNT decreased significantly and DPT also did not prolong in Huizhou City during the epidemic, and we believe these are due to the following: (1) all eight hospitals in Huizhou City have passed the certification of the National Brain Prevention and Treatment Hospital for Stroke; (2) have a designated channel for stroke patients, which is a new consensus that has been reached in the early stage of the outbreak
Fig. 4 Huizhou hospitals green channel process for stroke patients during the epidemic period
Triage nurse

1. Initiate the green channel for suspected acute stroke patients by conducting the FAST warning signs screening

2. Take body temperature; ask about contact exposure, travel history, and respiratory symptoms

\section{几}

Emergency doctor

1. Receive and screen patients, and repeat questions about contact exposure, travel history, and respiratory symptoms

2. Order head and chest CT, complete blood count, blood type, coagulation tests, basic metabolic panel, and troponin test

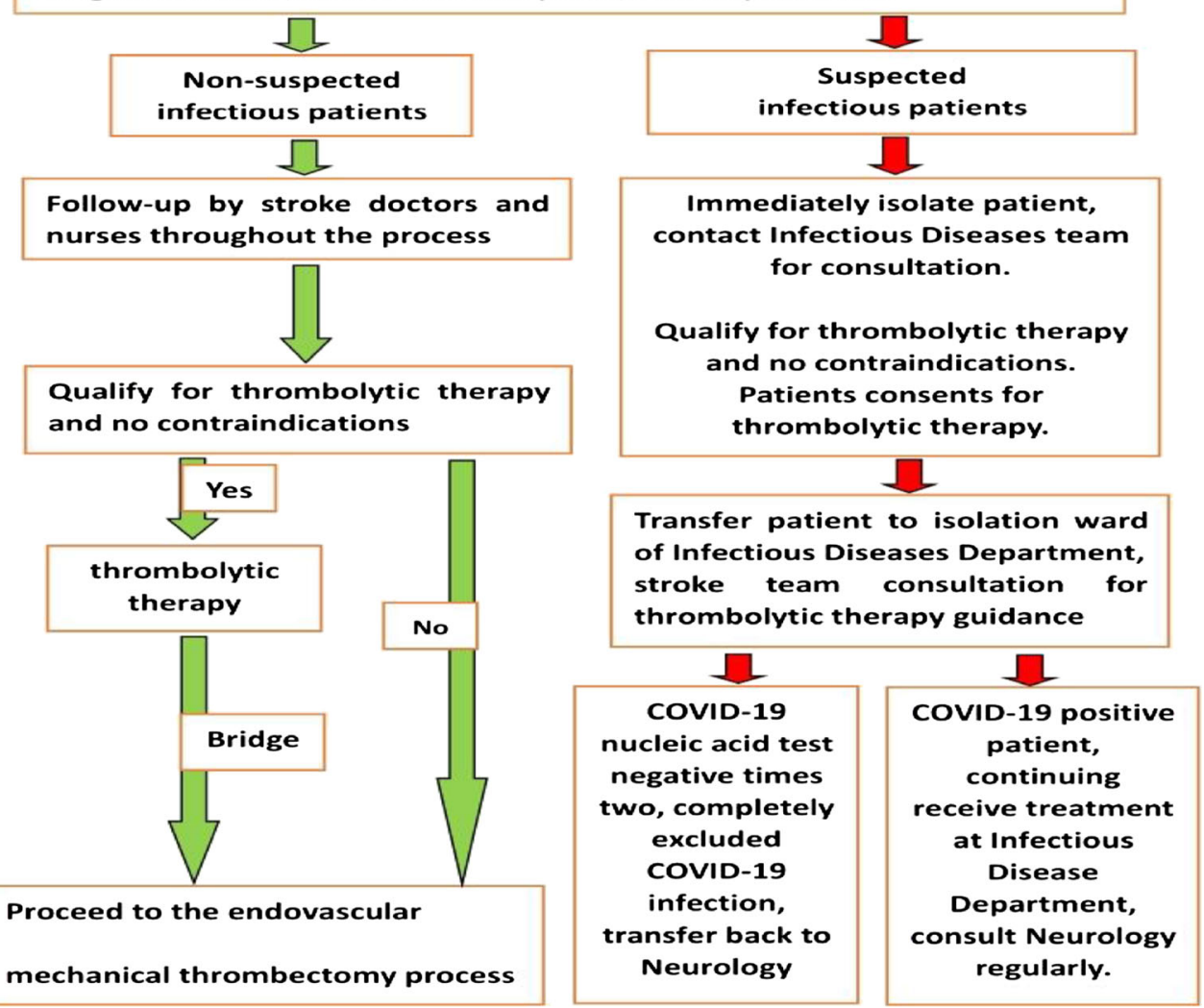


(see Fig. 4); (3) the closure of non-essential medical services decreased number of patients in the hospital; and (5) it is possible to centralize labor and resources to treat patients with acute ischemic stroke.

\section{Conclusion}

The number of acute ischemic stroke patients receiving reperfusion therapy significantly decreased during the COVID-19 pandemic compared with the same period in 2019, patients who visited the hospital were more severely ill, and the OPT time was longer. Regardless of the pandemic, self-visit remained as the main mode of transport for stroke patients to the hospital. However, for patients with moderate to severe stroke, most of them chose 120 rescue systems, whereas mild stroke patients chose to visit the hospital using their own transportation. Although prevention and control cycle of the COVID-19 pandemic in Huizhou City lasted about 5 weeks in 2020, the psychological impact still exists in our communities.

Supplementary information The online version of this article (https://doi. org/10.1007/s10072-020-04938-w) contains supplementary material, which is available to authorized users.

Acknowledgments This work was supported by the other 7 certified stroke reperfusion treatment hospitals in the Huizhou City of China.

\section{Compliance with ethical standards}

Ethical approval The study was approved by the Ethics Committee of Huizhou City Central Municipal Hospital 【KYLL2020120】. All procedures performed in studies involving human participants were in accordance with the ethical standards of the institutional and/or national research committee and with the 1964 Helsinki declaration and its later amendments or comparable ethical standards. Data were handled in a manner that did not reveal the identity of any patients or control subjects, so the form of consent (written/oral) for individual was not required.

Conflict of interest The authors declare that they have no competing interests.

\section{References}

1. Lan B, Lu P, Zeng Y, Li X, Ou X, Li J, Li H (2015) Clinical imaging research of the first Middle East respiratory syndrome in China. Radiol Infect Dis 2:173-176. https://doi.org/10.1016/j.jrid. 2015.11.004
2. Peng B, Wu B (2018) Chinese guidelines for dignosis and treatment of acute ischemic stroke 2018. Chin J Neurol 51:666-682. https:// doi.org/10.3760/cma.j.issn.1006-7876.2018.09

3. Li Q, Guan X, Wu P, Wang X, Zhou L, Tong Y, Ren R, Leung KSM, Lau EHY, Wong JY, Xing X, Xiang N, Wu Y, Li C, Chen Q, Li D, Liu T, Zhao J, Liu M, Tu W, Chen C, Jin L, Yang R, Wang Q, Zhou S, Wang R, Liu H, Luo Y, Liu Y, Shao G, Li H, Tao Z, Yang Y, Deng Z, Liu B, Ma Z, Zhang Y, Shi G, Lam TTY, Wu JT, Gao GF, Cowling BJ, Yang B, Leung GM, Feng Z (2020) Early transmission dynamics in Wuhan, China, of novel coronavirus-infected pneumonia. N Engl J Med 382:1199-1207. https://doi.org/10. 1056/NEJMoa2001316

4. Chen X, Tian J, Li G, Li G (2020) Initiation of a new infection control system for the COVID-19 outbreak. Lancet Infect Dis 20: 397-398. https://doi.org/10.1016/S1473-3099(20)30110-9

5. Ilzecka J, Stelmasiak Z (2000) Practical significance of ischemic stroke OCSP (Oxfordshire Community Stroke Project) classification. Neurol Neurochir Pol 34(1):11-22

6. Zhao J, Rudd A, Liu R (2020) Challenges and potential solutions of stroke care during the coronavirus disease 2019 (COVID-19) outbreak. Stroke. 51:1356-1357. https://doi.org/10.1161/ STROKEAHA.120.029701

7. Zhao J, Li H, Kung D, Fisher M, Shen Y, Liu R (2020) Impact of the COVID-19 epidemic on stroke care and potential solutions. Stroke. 51(7):1996-2001. https://doi.org/10.1161/STROKEAHA. 120.030225

8. Montaner J, Barragán-Prieto A, Pérez-Sánchez S, EscuderoMartínez I, Moniche F, Sánchez-Miura JA, Ruiz-Bayo L, González A (2020) Break in the stroke chain of survival due to COVID-19. Stroke. 51(8):2307-2314. https://doi.org/10.1161/ STROKEAHA.120.030106

9. Aguiar de Sousa D, Sandset EC, Elkind M (2020) The curious case of the missing strokes during the COVID-19 pandemic. Stroke. 51(7):1921-1923. https://doi.org/10.1161/STROKEAHA.120. 030792

10. Abdullah AS, Tomlinson B, Cockram CS, Thomas GN (2003) Lessons from the severe acute respiratory syndrome outbreak in Hong Kong. Emerg Infect Dis 9:1042-1045. https://doi.org/10. 3201/eid0909.030366

11. Medical group of Tongji Hospital Affiliated to Tongji Medical College of Huazhong University of science and technology. A quick guideline to diagnosis and treatment of pneumonia caused by new coronavirus(First edition). http://guide.medlive.cn/ guideline/19852, 2020-1-22

12. Huang C, Wang Y, Li X et al (2020) Clinical features of patients infected with 2019 novel coronavirus in Wuhan, China. Lancet 395(10223):497-506. https://doi.org/10.1016/S0140-6736(20) 30183-5

13. The Writing Group of the Expert Consensus for Clinical Prevention and Treatment of Coronavirus Disease 2019(COVID-19) for Neurologist. Expert consensus for clinical prevention and treatment of coronavirus disease 2019 (COVID-19) for neurologists, Chin J Neurol. 2020. 53(3): 166-170. https://doi.org/10.3760/cma.j.issn. 1006-7876.2020-03-002

Publisher's note Springer Nature remains neutral with regard to jurisdictional claims in published maps and institutional affiliations. 